\title{
Titration Design: An Important Key in Drug Dose Determination
}

\author{
R. Santosh Kumar and R Venkata Sandhya \\ GITAM Institute of Pharmacy, GITAM (Deemed To Be University) Rushikonda, Visakhapatnam-530045, Andhra Pradesh, India.
}

\begin{abstract}
Drug titration is the process of adjusting the dose of a medicine for the maximum benefits without adverse effects. To determine the optimum dose range the clinical trials are carried out and it is divided into two types of study design. The parallel type study design and titration type study design. The titration studies provide the information about the cumulative effects of the drug. Titration designs are mainly used in dose determining especially in insulin, anticonvulsants, anti-depressants, and sedatives. In the analysis of titration design, the phase I, II, III clinical trials are carried out to determine the dose-response relationship, safety and efficacy of the drug.
\end{abstract}

Keywords: Titration design, Dose, Response, Safety

Article Info: Received 11 June 2019; $\quad$ Review Completed 20 August 2019; Accepted 24 August 2019; $\quad$ Available online 30 August 2019

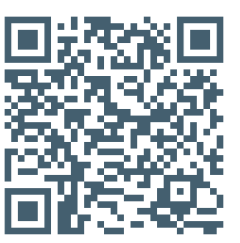

Cite this article as:

Santosh Kumar R, Venkata Sandhya R, Titration Design: An Important Key in Drug Dose Determination, Journal of Drug Delivery and Therapeutics. 2019; 9(4-s):826-828 http://dx.doi.org/10.22270/jddt.v9i4-s.3374

*Address for Correspondence:

R. Santosh Kumar, GITAM Institute of Pharmacy, GITAM (Deemed to be University), Gandhinagar, Visakhapatnam530045, Andhra Pradesh, INDIA.

\section{INTRODUCTION:}

For the development of a new drug, the dose range that provides the information about optimal therapeutic risk/benefit ratio in the targeted population to be determined .The clinical trials are carried out to determine the optimum dose range and it is divided into two types of study design.

The parallel groups, fixed dose study is the first type of design, and it is the simplest and most commonly used design to learn about the dose range .In parallel group study, the subjects are randomly allocated to receive one set of fixed doses for the entire study duration. Dose adjustments are not allowed and the subject receives only one dose level.

The dose titration study is the second type of design. In this type study, all subjects are started from the lowest set of dose levels. Subjects who respond on the low dose stay on that dose, and the other subjects who do not respond receive the next highest dose. This procedure is repeated until all subjects are drop out, respond, or reach the highest dose level.

\section{Overview:}

In the typical titration study, the response is based on some fixed criteria, the dose is increased with time and the subject may receive more than one dose level. Variations of typical titrations include placebo group, if the titration dose up or down, and a forced titration, in which all subjects receive higher doses despite of their responses at lower dose, and they do not drop for safety.

The difference between the titration and parallel study in a titration study is, if a subject fails to respond at a lower dose they receives the next higher dose, the target populations are different at each titration step. For example, in a parallel study the target population for subjects who receive dose level 2 is all subjects who have the disease. In a titration study, the target populations for subjects who receive the dose level 2 have the disease but failed to respond for the lower dose level 1.For titration study the target populations at dose level 2 are different in each study. This is the reason for the responders at a particular dose level in a parallel study should not be compared directly with the responders at that same level in a titration study. The analysis and interpretation of a titration study is more complicated than the parallel study. [1,2]

\section{Uses of Titration Designs}

\section{Clinical Rationale:}

In a titration study, the subjects are exposed to the amount of drug necessary to control their disease. This is the good clinical practice and is the main reason to titrate the dose. The titration studies provides the information on the sequential and cumulative effects of the drug, so they are used in clinical trials with drugs intended for long term use at varying dose levels such as antihypertensive drugs. 
Titration designs are used for diabetic subjects in evaluating formulations of insulin, because it is usually necessary that dose for each individual subject should be titrated up or down until the desired response is reached.

Even if titration appears to be appropriate for clinical reasons, the following requirements are necessary for the appropriate use of titration study.

- The response to the titration must be well-defined and easily measurable.[3]

- The number of dropout cannot be excessive. [3]

- The cumulative effect of the drug must be nominal. [3]

- If the response is irreversible, such as cure of a bacterial infection, then the use of a titration design is not appropriate. $[1,3]$

- Titrations design's may not be suitable in diseases such as ceteoporosis, Alzheimer's disease, or depression because the desired response takes long period of time if the treatment is extended it may be difficult to measure.

\section{Disadvantages of Titration:}

- During the course of treatment the disease may progress, the subjects may exhibit spontaneous improvement, or the drug may have some cumulative effects.

- One of the important issues in a titration study is deciding when to titrate the dose. If the dose is titrated upwards before its full effect is realized, the subject exposed to high dose level and the recommended is overestimated.

- With some drugs the carryover effect may exist from one dosing period to another, because in titration study the doses are given in increasing order, this carryover effect maybe harder to estimate compared to other types of designs.

\section{Advantages of Titration:}

- Titrations designs provide both practical and statistical advantages.

- The main practical advantages of titration designs are in compliance with good clinical practice, and the subjects are exposed only to the required amount of the drug to control their disease, and in the same dose the drug will be prescribed

- The statistical advantage is that each subject may receive more than one dose, titration design provide information on the distribution of individual dose response curves and population average dose response curve.

- Another statistical advantage is that titration studies can be used to examine the response over a wide range of doses, with smaller number of subjects than a parallel study with same statistical power.

- Sheiner et al stated that titration study can be more advantageous than the parallel or cross over study, if a patient specific parameter dose response model is used, based on comparison between the crossover, parallel, and titration designs in dose ranging studies.

- Titration designs are useful in phase I tolerance trials, the goal is to assess the safety of the drug. In early phase I/II exploratory studies, when the drug is assumed to have an effect but little is known about dose response relationship.[1,4]

- The goal of these studies is to examine the effectiveness of the drug over a wide range of doses to define the shape of the dose response curve.

- $\quad$ The benefit in this phase III studies is the subjects are more closely similar to the target population of subjects with the disease. With the incorporation of a placebo group and appropriate analysis and it provides pieces of evidence of the safety and overall efficacy of the drug and effectiveness of the various doses.[5]

\section{Analysis of a Titration Design:}

\section{Types of Analysis}

\section{Life Table Approach: [1]}

Based on the analysis of a titration study three general assumptions are made.

1. During the period, one dose which is administered is long enough for the physiological systems to stabilize.

2. Subject who responds at one dose will respond at all higher doses.

3. The number of subjects who receive a particular dose, the number of responders at that dose follows a binomial distribution.

\section{Incomplete Design Approach:}

In a complete design, all subjects are exposed to all dose levels. In incomplete design all subjects are not exposing to all dose levels. The assumption is that a subject who responds at one dose may not at all higher doses in the incomplete design approach.

\section{Contingency Table Approach:}

In phase I/II clinical trials the dose-response relationship is established. In phase III clinical trials, efficacy and safety are confirmed. A phase III clinical trials can be used to determine, the starting dose which is therapeutically useful, based on overall response rate and a maximally effective dose is reached whether it titrates to the low or high dose.

\section{Titration with Placebo Controls:}

If a placebo effect is expected to be high the magnitude of the effect and efficacy of the drug can be estimated by the insertion of the placebo control group (it contains all the excipients except drug). The effect of the dose can be measured by including the placebo controls for that dosing group.

\section{Analysis of Safety Data:}

The analysis of safety data in a titration study presents special problems, and the estimation of the risky/benefit ratio is challenging, even the response is easily measurable and occurs in a short period of time. The incidence of the adverse effects depends upon the amount of the dose given to the subject.

The assumption is that analyzing the safety data in titration study is different for analyzing the efficacy data. The assumption is that analysis of efficacy data in a titration study is that a subject who responds at lower doses will respond at all higher dose levels. If a subject experiences an adverse effect at one dose level and it is not necessary that subject would have same adverse effects at higher dose levels. 


\section{CONCLUSION:}

Parallel and titrations studies are used in dose finding and comparative studies. Parallel studies are useful in determining the effectiveness of various doses through the use of independent dose groups having the same duration of treatment and fixe dose, short term prescriptions. Titrations studies provide information about the sequential and cumulative effects of the drug at various dose levels, and used for long term prescriptions. [5]

The titration designs can provide information on the safety and efficacy of the drug. By including a placebo group and taking into account the dose, speed, and time of the titration, and the titration design, it can provide the information about the effect by dose, but the result is different for each subject with variation of time.

\section{REFERENCES:}

1. Chuang, C. The analysis of a titration study. Stat. Med 1982, 6, 583-590.

2. Pun, E.F.C. A parallel dose titration design to determine dose range, ASA, Pro Biophys 1990, 159-164.

3. JCH Harmonized Tripartite Guideline, E-4. In Dose-Response Information Support Drug Registration, 1994.

4. Chuang-Stein, C. Two approaches for the analysis of a titration study with a placebo control. Commun Stat, Theory Methods 1988, 17, 821-832.

5. Shih, W.J; Gould, AL; Hooang, IK, The analysis of titration studies in phase III clinical trials, Stat. Med.1989, 8,583-591. 